Western University

Scholarship@Western

Department of Economics Research Reports

Economics Working Papers Archive

1993

\title{
Monopolistic Competition, Increasing Returns and Self-Fulfilling Prophecies
}

Brian Rivard

Follow this and additional works at: https://ir.lib.uwo.ca/economicsresrpt

Part of the Economics Commons

Citation of this paper:

Rivard, Brian. "Monopolistic Competition, Increasing Returns and Self-Fulfilling Prophecies." Department of Economics Research Reports, 9308. London, ON: Department of Economics, University of Western Ontario (1993). 


\title{
RESEARCH REPORT 9308
}

\section{Monopolistic Competition. Increasing Returns and Self-Fulfilling Prophecies}

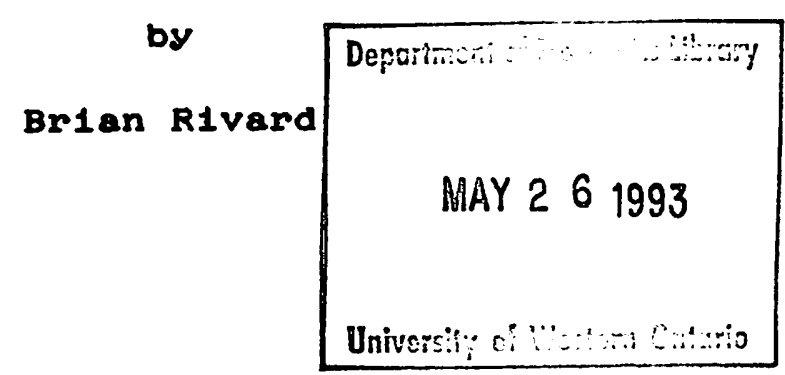

November 1992

\author{
Department of Economics \\ Social Science Centre \\ UnIvereity of Western Ontario \\ London, Ontar1o, Canada
}

N6A $5 \mathrm{C2}$ 


\title{
Monopolistic Competition, Increasing Returns and Self-Fulfilling Prophecies
}

\author{
Brian Rivard* \\ The University of Western Ontario, \\ London Ontario, Canada \\ N6A $5 \mathrm{C} 2$
}

November 1992

\begin{abstract}
This paper constructs a simple overlapping generations model with money and production in an environment of monopolistically competitive firms and an increasing returns to scale production technology. Within this framework symmetric stationary sunspot equilibria are generated without requiring the assumption of gross complementarity between future consumption and current leisure. Output fluctuations may also be highly persistent in this model. Journal of Economic Literature Classification Number: E32.
\end{abstract}

-I would like to thank, without implicating, the following people for helpful comments and criticisms on earlier drafts of this paper: Murali Agastya, Scott Hendry, Dan Peled and Steve Williamson. I would also like to thank Karl Shell and an associate editor for their informative review. I am especially grateful to Peter Howitt for many valuable conversations. 


\section{Introduction}

There has been a recent interest in the notion that extraneous uncertainty could be a factor in generating economic fluctuations. Equilibria in which this occurs are often referred to as sunspot equilibria. Some of the early research in this area included Shell [15], Azariadis [2!, Cass and Shell [5], Spear [18], and Azariadis and Guesnerie [3]. Much of this research concentrated on finding conditions for the existence of sunspot equilibria in an overlapping generations (OG) economy.

A particularly notable contribution to this literature was Azariadis [2]. Azariadis constructed an OG model with money and production and found that a necessary condition for the existence of sunspot equilibria is that future consumption and current leisure be gross complements, i.e. the supply of labour schedule must be backward bending. Azariadis also concluded that in a model where the price level follows a simple two state markov process, the existence of sunspot equilibria implies the stationary transition probabilities associated with the markov process sum to less than 1 . This would suggest a low level of persistence in aggregate output.

Although Azariadis demonstrated the existence of sunspot equilibria, the afore mentioned conditions raise some doubt as to the importance of such equilibria. The main concern is that the conditions of gross complementarity and a low level of persistence in output are not well supported empirically. For instance, to achieve a backward bending supply schedule, the income effect of a wage increase must dominate the substitution effect. However, in most empirical studies on labour supply, this is shown not to be the case. ${ }^{1}$ Furthermore, researchers such as Campbell and Mankiw [6] and Phillips [14] suggest that aggregate output contains a unit root which would imply output fluctuations are highly persistent.

For these reasons, a number of authors have tried to find a more satisfactory environment for which sunspot equilibria could exist. This analysis generally relies on the presence of 
market frictions to prove existence. For instance, Howitt and McAfee [10] derive sunspot equilibria in a model with transactions externalities, Woodford [21] creates sunspot equilibria by using borrowing constraints in an infinitely lived agents model, Chatterjee, Cooper and Ravikumar [7] develop an economy with strategic complementarity in entry decisions to derive sunspot equilibria, Weil [20] demonstrates the possible existence of sunspot equilibria in a model with an increasing returns to scale storage technology. None of these works require the above mentioned assumptions.

The purpose of the present paper is to show that stationary sunspot equilibria can exist in an OG model without requiring the assumptions of gross complementarities and a low level of persistence in output. The usual simple OG model with money and production is presented with the exception of there being monopolistic competition in the product market and increasing returns to scale in the production technology.

That a model with monopolistic competition in the product market and increasing returns to scale in the production technology can give rise to sunspot equilibria should not be surprising. Such a result is consistent with what Shell [16] refers to as the Philadelphia folk theorem; models that incorporate market friction through which the existence of non-optimal equilibria is made possible, also admit the existence of sunspot equilibria. As mentioned, the works by Howitt and McAfee; Chatterjee, Cooper and Ravikumar; Woodford and Weil, all rely on market frictions to demonstrate the existence of sunspot equilibria. This is consistent with Shell's conjecture. Other recent works for which the presence of market frictions leads to the existence of sunspot equilibria are Shell and Wright [17] Which examines an economy with nonconvex consumption sets and Aumann, Peck and Shell 1 , who give examples of sunspot equilibria in a model with asymmetric information.

Models with market frictions similar to those used in the present paper can be found in Kehoe, Levine and Romer [12], and Spear [19!, which introduce a production externality in a dynamic capital accumulation model as well as Peck and Shell [13], which examine an 
imperfectly competitive economy. These articles show that as a result of introducing such market frictions, previously determinate equilibria become indeterminate; sunspot equilibria can exist.

For the present model, the presence of monopolistic competition and increasing returns to scale leads to the possible existence of multiple steady state equilibria. These steady state equilibria can be Pareto ranked. The existence of multiple steady state equilibria allows for the generation of sunspot equilibria as randomizations between allocations in the neighborhood of these steady states. Such sunspot equilibria do not rely on the assumption of gross complementarities, nor do they lead to a low level of persistence in output.

The remainder of the paper will proceed as follows. Section 2 presents the model and discuses the agent's problem as well as defines a perfect foresight equilibrium. Section 3 examines the possible existence of multiple equilibria. Section 4 defines and constructs sunspot equilibria as randomizations between allocations in the neighborhood of steady state equilibria. Section 5 gives some summary remarks.

\section{The Model}

Consider an overlapping generations economy populated by a continuum of two period lived agents born in each period. Normalize the size of the population to 1 . Time is discrete in this economy with $t=0,1, \ldots \infty$. Each agent $j$ is endowed with one unit of leisure and possesses a technology from which output can be produced. Agent $\mathrm{j}$ can only produce a type j consumption good, but is free to consume the goods of all other agents. The technology at date $\mathrm{t}$ is given by $y_{t}^{j}=f\left(n_{t}^{j}\right)$ where $y_{t}^{j}$ denotes agent $j$ 's output at date $t, n_{t}^{j}$ denotes the level of labour used by agent $\mathrm{j}$ in time $\mathrm{t}$ production, and $f(\cdot)$ is an increasing returns to scale production function; i.e. $f \prime(\cdot)>0, f \prime \prime(\cdot)>0$. There are also a continuum of initial old, each endowed with 1 unit of fiat money.

To introduce monopolistic competition to the model, we follow Blanchard and Kiyotaki [4] 
by adopting constant elasticity of substitution (CES) specifications in utility. It is assumed that utility is additively separable in consumption and leisure, with agents producing when young and consuming when old. The preferences of each agent are thus given by:

$$
V\left(c_{t+1}^{j}, n_{t}^{j}\right)=u\left(c_{t+1}^{j}\right)-g\left(n_{t}^{j}\right)
$$

where $c_{t+1}^{j}=\left[\int_{0}^{1}\left(c_{t+1}^{j}(i)\right)^{\frac{\theta-1}{\theta}} d i\right]^{\frac{\theta}{\theta-1}}$ is a CES consumption bundle, $c_{t+1}^{j}(i)$ is the consumption of good $i$ by agent $j$ at date $t+1$, and the parameter $\theta$ is the elasticity of substitution between goods in utility. ${ }^{2}$ For notational convenience, define $k(y) \equiv g\left(f^{-1}(y)\right)$ with $f^{\prime-1}(y) \geq 0$ and $f^{\prime \prime-1}(y) \leq 0$. The standard concavity, differentiability and continuity conditions are imposed on $u(\cdot)$ and $-g(\cdot)$ with $g^{\prime}(y) \rightarrow 0$ as $y \rightarrow 0 ; g^{\prime}(y) \rightarrow \infty$ as $y \rightarrow f(1) ; g^{\prime \prime}(y)>0 ; u \prime(y) y<\infty$ as $y \rightarrow 0$; There is also the following assumption imposed on the production function.

Assumption 1: $f f^{-1}(y) y<\infty$ as $y \rightarrow 0 ; f^{-1}(0)=0 .^{3}$

Finally, define $P_{t}=\left[\int_{0}^{1}\left(p_{t}(i)\right)^{1-\theta} d i\right]^{\frac{1}{1-\theta}}$ as the nominal price index at date $t$. Since there is a continuum of agents, each agent taking the actions of others as given is equivalent to taking $P_{t}$ as given.

\subsection{The Agent's Problem}

Agent j's problem is then to choose levels of $c_{t+1}^{j} ; y_{t}^{j} ; n_{t}^{j} ; p_{t}^{j}$, so as to maximize his utility subject to a budget constraint and the constraints on technology. The agent takes the nominal price index as given and faces a downward-sloping demand schedule for his product that is derived from the optimization problems of the other agents. The budget constraint is given by;

$$
p_{t}^{j} y_{t}^{j}-\int_{0}^{1} p_{t+1}(i) c_{t+1}^{j}(i) d i=0
$$

where $p_{\ell}^{j}$ is the price of agent $\mathrm{j}^{\prime}$ s product at time $t$. The technology constraints are

$$
y_{t}^{j}=f\left(n_{t}^{j}\right)
$$


and

$$
0 \leq n_{t}^{j} \leq 1
$$

Finally, the demand curve for agent j's product is,

$$
y_{t}^{j}=\frac{\left(p_{t}^{j}\right)^{-\theta}}{P_{t}^{1-\theta}}
$$

It should be noted that the CES specification in utility implies each agent will face a constant elasticity of demand for his product and sell his product at a constant markup of price over marginal cost. This result can be derived directly from the demand curve for agent $j$ 's product. Clearly the elasticity of demand is $\frac{d y_{t}}{d p_{t}} \cdot \frac{p_{t}}{y_{t}}=-\theta$ which is constant. Furthermore, marginal revenue is given by $\frac{d p_{t}}{d y_{t}} y_{t}+p_{t}=p_{t}\left[\frac{\theta-1}{\theta}\right]$. Equating marginal revenue and marginal cost and rearranging terms implies $p_{t}=\left[\frac{\theta}{\theta-1}\right] M C$. The term $\left[\frac{\theta}{\theta-1}\right]$ represents the constant markup coefficient.

\subsection{A Perfect Foresight Equilibrium}

The solution to the agents problem is derived in appendix A. From the necessary first order conditions for an optimum we have;

$$
k \prime\left(y_{t}^{j}\right)=u\left(\left(c_{t+1}^{j}\right)\left[\int_{0}^{1}\left(p_{t+1}(i)\right)^{1-\theta} d i\right]^{\frac{1}{\theta-1}} p_{t}^{j}\left[\frac{\theta-1}{\theta}\right]\right.
$$

To simplify the analysis, we can focus on symmetric equilibria. In a symmetric monetary equilibrium it must be that $p_{t} y_{t}=1$ (i.e. the money market clears). Thus ( 1 ) implies;

$$
k \prime\left(y_{t}\right) y_{t}=\left[\frac{\theta-1}{\theta} ; u\left(y_{t+1}\right) y_{t+1}\right.
$$

Equation ( 2) simply suggests that each agent will maximize his welfare by equating his marginal benefit from production to his marginal cost from work. This is similar to the first order necessary condition derived by Azariadis. In Azariadis, agents maximizing welfare implied the equation $u /\left(y_{t+1}\right) y_{t+1}=y_{\ell} g\left(y_{t}\right)$ must be satisfied for all $t$. However, in the 
case of the present model, the addition of the markup coefficient reflects the fact that the monopolistically competitive firm is able to sell his product at a higher price. Furthermore, the marginal cost of labour function now includes the effects of the increasing returns to scale production technology. It is this difference that allows for the possible existence of multiple steady states. This issue shall be examined at further length in the ensuing section.

It should be noted that for equation (2) to represent a maximum solution, the second order necessary conditions must be satisfied. The second order necessary condition for an optimum is:

$$
u \prime \prime(\cdot)\left(\frac{p_{t}}{p_{t+1}}\right)^{2}\left[\frac{1-\theta}{\theta}\right]^{2}+\frac{u \prime(\cdot)}{\theta p_{t+1} y_{t}^{2}}\left[\frac{1-\theta}{\theta}\right]-k \prime \prime\left(y_{t}\right) \leq 0
$$

For notational purposes we will refer to this condition as (S.O.C) $\leq 0$.

Since our interest is in steady state equilibria, we can evaluate (3) at the steady state optimum. It can be shown that the second order necessary condition evaluated at a steady state optimum is satisfied ${ }^{4}$ iff:

$$
\theta\left[\varepsilon_{u}-\varepsilon_{k}\right]-\left[\varepsilon_{u}+1\right] \leq 0
$$

where $\varepsilon_{u}$ is the elasticity of the marginal utility of consumption and $\varepsilon_{k}$ is the elasticity of the inverse of the marginal product of leisure plus the elasticity of the disutility of leisure. By assumption $\varepsilon_{u}<0$ and $\varepsilon_{k} \quad \lessgtr 0$. For what follows, it will also be assumed that $\varepsilon_{u}>-1$. This is the assumption that consumption and leisure be gross substitutes. ${ }^{5}$

With this we are now able to define a perfect foresight symmetric monetary equilibrium (PFSME). A PFSME is a pair of sequences $\left(p_{t}\right)_{t=0}^{\infty},\left(y_{t}\right)_{t=0}^{\infty}$ such that;

i) equation (2) and ( 3 ) are satisfied for all $t \geq 1$

ii) $p_{t} y_{t}=1$ for all $\mathrm{t}$

iii) $y_{0} \in[0, f(1)]$; i.e. the initial level of output is feasible.

In the following section, our focus will be in finding the conditions for which multiple steady state equilibria exist. It is necessary that our candidate equilibria satisfy equation 
4 above. However, satisfying equation 4 (along with equation 2 ) will only guarantee the existence of a local maxima. To assure the existence of a global maxima, we will assume that the objective function of an agent, given the actions of the other agents, is concave. That is, the second order derivative of the function $V(y ; P)=u\left(y^{\frac{\theta-1}{\theta}} P^{\frac{-1}{\theta}}\right]-k(y)$ is nonpositive, where $\mathrm{P}$ is the steady state price index representing the actions of all other agents. More explicitly,

Assumption 2: $u \prime \prime(\cdot)\left[\frac{\theta-1}{\theta}\right]^{2} y^{\frac{-2}{\theta}} P^{\frac{-2}{\theta}}-\frac{w(\cdot)}{\theta}\left[\frac{\theta-1}{\theta}\right] P^{\frac{-1}{\theta}} y^{\frac{-(\theta+1)}{\theta}}-k \prime \prime(y) \leq 0$ for all $y \in[0, f(1)]$.

It is now possible to characterize the conditions under which multiple equilibria may exist.

\section{The Existence of Multiple Steady State Equilibria}

As was mentioned, the presence of monopolistic competition and increasing returns to scale in an OG model creates the possibility of the existence of multiple steady state equilibria. To illustrate this possibility for the present model, define the function $z(y)=\left[\frac{\theta-1}{\theta}\right] u /(y) y-k \prime(y) y$. Then if $y$ is a steady state equilibrium, it must satisfy $z(y)=0$.

Furthermore, by assumption 1 and the assumptions on preferences we have, $z(y) \geq 0$ as $y \rightarrow 0$ and $z(y) \leq 0$ as $y \rightarrow f(1)$. Therefore $z \prime(y)$ must be nonpositive for at least some values of $y \in[0, f(1)]$. However, since $z \prime(y)=\left[\frac{\theta-1}{\theta}\right] u \prime(y)\left[1+\varepsilon_{u}\right]-k \prime(y)\left[1+\varepsilon_{k}\right] \frac{\leq}{>}, z(y)$ may also be upward sloping for some $y \epsilon[0, f(1)$. Note, at a steady state equilibrium, $z \prime(y) \geq 0$ if and only if $\varepsilon_{u} \geq \varepsilon_{k}$ and $z \prime(y) \leq 0$ if and only if $\varepsilon_{u} \leq \varepsilon_{k}$. With this information, it is possible to graph various potential configurations of the function $z(y)$. This is done in figure 1 .

In figure la, $z(y)$ is constructed so that there exists three values of $y$ with $z(y)=0$. These three values; $y^{*}, y^{\mu *}$, and $y^{\cdots \cdots}$ represent possible steady state equilibria. ${ }^{6}$ In figure $1 \mathrm{~b}, z(y)$ is constructed so that $y^{*}$ is the only equilibrium. What makes multiple steady state equilibria possible in figure $1 \mathrm{a}$ and not in figure $1 \mathrm{~b}$, is the fact that at $y^{*-}, z(y)$ is positively sloped. Since $z(0) \geq 0$ and $z(f(1)) \leq 0$, it then must be that $z(y)=0$ for at least one value of $y$ 


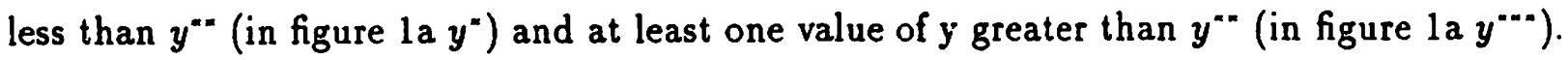
Furthermore, at $y^{*}$ and $y^{* \cdots}$, the second order condition for an optimum is satisfied, hence $y^{*}$ and $y^{*-*}$ represent steady state equilibria. To state more formally a sufficient condition for the existence of multiple steady state equilibria we offer the following proposition.

Proposition 1: Multiple steady state equilibria exist if there exists a $\bar{y} \epsilon^{\circ} 0, f(1)^{\prime}$. such that;

i) $z(\bar{y})=0$

ii) $\varepsilon_{u}(\bar{y})>\varepsilon_{k}(\bar{y})$

Proof: Let $\bar{y} \epsilon[0, f(1)]$ satisfy i) and ii). Let $\delta>0$ be an arbitrarily small real number. Condition ii) implies $z \prime(\bar{y})>0$. But then i) implies $z(\bar{y}-\delta)<0$ and $z(\bar{y}+\delta)>0$. Furthermore, we have $z(0) \geq 0$ and $z(f(1)) \leq 0$. Therefore, there must exist a $y^{*}<\bar{y}$ such that $z\left(y^{*}\right)=0$ and $\varepsilon_{u}\left(y^{*}\right)<\varepsilon_{k}\left(y^{*}\right)$ and a $y^{\cdots *}>\bar{y}$ such that $z\left(y^{\cdots-}\right)=0$ and $\varepsilon_{u}\left(y^{\cdots-}\right)<\varepsilon_{k}\left(y^{\cdots *}\right)$. Hence $y^{*}$ and $y^{x-*}$ both represent steady state equilibria. Q.E.D.

At this point, it is interesting to examine the significance of increasing returns and monopolistic competition for the existence of multiple steady state equilibria. First, if instead of exhibiting increasing returns to scale, the production technology exhibited constant returns to scale, the function $z(y)$ would be written as $z(y)=\left[\frac{\theta-1}{\theta} ; u(y) y-g(y) y\right.$, so that $z \prime(y)=\left[\frac{\theta-1}{\theta}\right] u \prime(y)\left[1+\varepsilon_{\mathrm{u}}\right]-g \prime(y)\left[1+\varepsilon_{g}\right]<0$. In this case $z(y)$ could only be negatively sloping since, by the assumption on preferences, $\varepsilon_{u}(y)<\varepsilon_{g}(y)$ for all $y$. But then an equilibrium such as $y^{x \approx}$ in figure la could never exist in the constant returns to scale economy. Therefore, in the present model, the assumption of increasing returns to scale is crucial for the existence of multiple steady state equilibria.

Secondly, if the market structure was perfectly competitive, the second order condition for an optimum evaluated at a steady state y, would be satisfied if and only if $\varepsilon_{u}(y) \leq \varepsilon_{k}(y)$. 
This would then tend to rule out points such as $y^{*-}$ in figure la as equilibria since the second order condition for an optimum would be violated at $y^{2=8}$

It is also possible to comment on the local stability of the steady state equilibria $y^{*}, y^{* *}, y^{* * *}$. For this economy, the law of motion can be written as $\frac{d y_{t}}{d y_{t+1}}=\frac{u\left(y_{t-1}\right) \cdot \frac{\theta-1}{\theta}\left[\left(1+e_{z}\left(y_{t+1}\right)\right]\right.}{k\left(y_{t}\right)\left(1+e_{k}\left(y_{t}\right)\right]}$. For steady state optima such as $y^{*}$ and $y^{\cdots}$ we have $\frac{d y_{i}}{d y_{t+1}}<1$ since $\varepsilon_{k}>\varepsilon_{u}$ at $y^{*}$ and $y^{\cdots \cdots}$. Thus equilibria such as $y *$ and $y \cdots$ will be locally unstable.

For equilibria such as $y^{*-}$, if $1+\varepsilon_{k}>0$, then $\frac{d y_{1}}{d y_{t+1}}>1$ and hence $y^{* *}$ will be locally stable. This follows from the fact that $\varepsilon_{k}<\varepsilon_{u}$ at $y^{* *}$. If $1+\varepsilon_{k}<0$, then $y^{* *}$ will be locally stable if $\varepsilon_{u}+\varepsilon_{k}>-2$. However, if $\varepsilon_{u}+\varepsilon_{k}<-2, y^{--}$will be locally unstable. ${ }^{9}$

As a further note, in relation to Azariadis and Guesnerie (1986), periodic equilibria of order two ( 2 cycles) may exist in this model, depending on the particular stability properties of the equilibrium law of motion. For instance, if $1+\varepsilon_{k}\left(y^{* *}\right)>0$, then the equilibrium law of motion would be positively sloped for all feasible values of $y_{0}$ and in particular, it would be locally stable at $y^{*-}$. In this instance, 2 cycles could not exist as a positively sloped law of motion would rule out the possibility of any oscillation in an equilibrium output path (i.e. either $y_{0} \geq y_{1} \geq \ldots \geq \bar{y}$ or $y_{0} \leq y_{1} \leq \ldots \leq \bar{y}$; where $\bar{y}$ represents a steady state equilibrium.) If on the other hand $1+\varepsilon_{k}\left(y^{\mu-}\right)<0$ a 2 cycle would exist only when $\epsilon_{k}\left(y^{\mu \prime}\right)+\epsilon_{u}\left(y^{*-}\right)<-2$ (i.e. $y^{* *}$ is locally unstable). In this case the law of motion would be sufficiently backward bending so as to generate the possibility of oscillation in the equilibrium output path. Further comment regarding 2 cycles and their relation to sunspot equilibria will be given in the following section.

In this economy, increasing returns to scale and monopolistic competition are also significant features for the existence of sunspot equilibria. As shall be seen in the following section, the existence of steady state equilibria such as $y^{* *}$ are required for the existence of sunspot equilibria in which leisure and consumption are gross substitutes. As was stated above steady state equilibria such as $y^{* *}$ exist in this economy because of increasing returns 
to scale and monopolistic competition.

\subsection{Numerical Example}

The following is a numerical example of an OG model in which consumption and leisure are gross substitutes and multiple steady state equilibria exist. Consider the following economy: $u(y)=\frac{y^{1-\gamma}}{1-\gamma}, g(y)=.25 y^{8}-.25 y^{4}+.65 y^{2} ; f^{-1}(y)=\frac{\alpha y^{1-\alpha}}{1-\alpha}$ with $\gamma=.06$ and $\alpha=.5$.iote, $f(n)=n^{2}$ and $f(\lambda n)^{2}=\lambda^{2} n^{2}=\lambda^{2} f(n)$ for $\lambda>1$. Hence $f(\cdot)$ exhibits increasing returns to scale. All agents are identical for all $t$.

The problem at date $t$ is thus;

$$
\max _{y} \Lambda=\frac{\left[\frac{p_{t}}{P_{t+1}} y_{t}\right]^{.94}}{.94}-.25 y_{t}^{4}+.25 y_{t}^{2}-.65 y_{t}
$$

The first order condition is thus;

$$
\left[\frac{p_{t}}{P_{t+1}}\right]^{-.06}\left[\frac{\theta-1}{\theta}\right]-y_{t}^{3}+.5 y_{t}-.65=0 .
$$

Letting $\theta=2$ it is easy to verify that there exists multiple equilibria. Solving the first order condition for the steady state relative optima yields the solutions, $y=.0154 ; y=.2355$; $\mathrm{y}=.4872$. The second order condition is given by $-.265 y^{-1.06}-3 y^{2}+.5 \leq 0$. It is easy to show that all three candidate equilibria satisfy this condition. Furthermore, we have

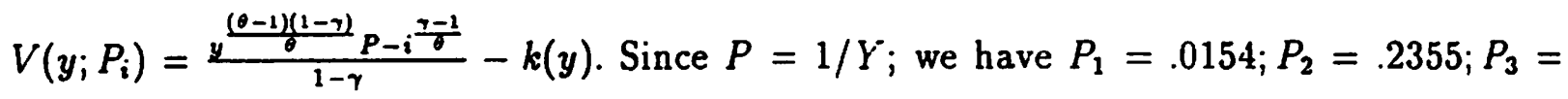
.4872. Therefore, after the proper substitution of parameter values we have,

$$
V \prime \prime\left(y ; P_{i}\right)=.2491 A_{i} y^{-1.53}-3 y^{2}+.5 \text { foralli }=1,2,3
$$

where $A_{1}=.1496, A_{2}=.5391, A_{3}=.7587$. It can be shown that $V \prime \prime(. ;)<$.0 for all $\mathrm{i}$ and for all feasible $y$ so that assumption 2 is satisfied. It is also easy to show that the optima can be Pareto ranked with $\Lambda(.4872)>\Lambda(.2355)>\Lambda(.0154) .{ }^{10}$ 


\section{Sunspot Equilibria}

To show the existence of a sunspot equilibrium (SE), we must first introduce extraneous uncertainty into the model. Let $I_{t}$ be the information set available to all agents at date $t$; $I_{t}$ contains all historical elements in the economy up until and including date t. Let $s_{t}$ be a particular element of $I_{t}$. Therefore, following the equilibrium concept of section 1 ; a pair of sequences of random variables $\left(p_{t}\right)_{t=0}^{\infty}\left(y_{t}\right)_{t=0}^{\infty}$ is a $S E$ if:

i) $E\left[u /\left(y_{t+1}\right) y_{t+1}\left[\frac{\theta-1}{\theta}\right] \mid I_{t}\right]=k \prime\left(y_{t}\left(s_{t}\right)\right) y_{t}\left(s_{t}\right)$

ii) $E\left[u \prime \prime(\cdot)\left(\frac{p_{t}\left(s_{t}\right)}{p_{t+1}}\right)^{2}\left[\frac{\theta-1}{\theta}\right]^{2}-\frac{u(\cdot)}{\theta p_{t}+1 y_{t}^{2}\left(o_{t}\right)}\left[\frac{\theta-1}{\theta} ; I_{t}\right]-k \prime \prime\left(y_{t}\left(s_{t}\right)\right) \leq 0\right.$

iii) $p_{t}\left(s_{t}\right) y_{t}\left(s_{t}\right)=1$ for all $s_{t}$.

iv) $y_{0} \in[0, f(1)]$

Because our interest is in finding SE that do not rely on the assumption of gross complementarity, we follow Azariadis by limiting ourselves to solutions with the markov property. That is ones for which $I_{t}=y_{t}$. In particular let $q_{i}=\operatorname{Pr}\left(y_{t+1}=y_{i} \mid \quad y_{t}=y_{i}\right)$ for $\mathrm{i} \in(1,2)$ so that output follows a two state markov process. Also define $\mathrm{W}\left(y_{t+1}\right)=\left[\frac{\theta-1}{\theta}\right] u\left(y_{t+1}\right) y_{t+1}$ and $K\left(y_{t}\right)=k \prime\left(y_{t}\right) y_{t}$. Focusing on symmetric equilibria, a symmetric stationary sunspot equilibrium (SSSE) can then be defined as a $\left(q_{1}, q_{2}, y_{1}, y_{2}\right)$ such that;

i) $q_{1} W\left(y_{1}\right)+\left(1-q_{1}\right) W\left(y_{2}\right)=K\left(y_{1}\right)$

ii) $\left(1-q_{2}\right) W\left(y_{1}\right)+q_{2} W\left(y_{2}\right)=K\left(y_{2}\right)$

iii) $\left.\left.\frac{\theta-1}{\theta^{2}}\left[q_{i}: W\left(y_{i}\right)\left[y_{i} \theta-1\right]-\theta W^{\prime}\left(y_{i}\right) !+\left(1-q_{i}\right) y_{j} . W^{\prime}\left(y_{j}\right) ! y_{j} \theta-1\right]-\theta W\left(y_{j}\right)\right]\right]-K^{\prime}\left(y_{i}\right) \leq 0$

$$
i, j=1,2 \quad i \neq j
$$

iv) $q_{i} \in[0,1]$ and $y_{i} \in[0, f(1)] ; \quad i \in(1,2)$

Conditions (i), (ii) and (iii) assure the local maximization of expected utility where (iii) represents the second order condition. Condition (iv) assures that $q_{i}$ and $y_{i}$ are feasible. Note, conditions (i) and (ii) are equivalent to;

$$
q_{1}=\frac{W\left(y_{2}\right)-K\left(y_{1}\right)}{W\left(y_{2}\right)-W\left(y_{1}\right)}
$$




$$
q_{2}=\frac{K\left(y_{2}\right)-W\left(y_{1}\right)}{W\left(y_{2}\right)-W\left(y_{1}\right)}
$$

Furthermore, $W \prime(y)>0$ so that consumption and leisure are gross substitutes. ${ }^{11}$

To illustrate the possible existence of SSSE we follow Howitt $\left[99_{j}\right.$ and Howitt and Mcafee [10] and generate SSSE as randomizations between multiple steady state equilibria. The benefit of constructing SSSE as randomizations between multiple steady state equilibria is that both the stationary transition probabilities $q_{1}$ and $q_{2}$ can be shown to be close to one in value. This contradicts the required assumption made in Azariadis that $q_{1}+q_{2} \leq 1$. As stated, this assumption implies a low level of persistence in output. However, in the present model, because $q_{1}$ and $q_{2}$ can be close to one, SSSE can be generated in an economy in which output fluctuations are highly persistent.

With this in mind, the objective of the following exercise is to show that given a $\left(y_{1}, q_{1}\right)$ and a $\left(y_{2}, q_{2}\right)$, there will be solutions $\bar{y}$ and $\dot{y}$ to the agent's maximization problem such that $\bar{y}=y_{1}$ and $\dot{y}=y_{2}$. Since the model may posses steady state equilibria such as $y^{*}$ and $y^{\cdots-}$ from figure 1a, we know there are solutions $\bar{y}=y^{*}$ and $\dot{y}=y^{\cdots *}$ with $q_{1}=q_{2}=1$. The implicit function theorem, if satisfied, can then be applied to find solutions for which $q_{1}<1$ and $q_{2}<1$. This solution will represent a SSSE. Therefore, to achieve this objective, define;

$$
\begin{aligned}
& \bar{y}\left(y_{1}, y_{2}, q_{1}\right) \equiv \operatorname{argmax}_{<y>} \quad q_{1} u\left(y^{\frac{\theta-1}{\theta}} y_{1}^{\frac{1}{\theta}}\right)+\left(1-q_{1}\right) u\left(y^{\frac{\theta-1}{\theta}} y_{1}^{\frac{1-\theta}{\theta}} y_{2}\right)-k(y) \\
& \grave{y}\left(y_{1}, y_{2}, q_{2}\right) \equiv \operatorname{argmax}_{<y>} \quad q_{2} u\left(y^{\frac{\theta-1}{\theta}} y_{2}^{\frac{1}{\theta}}\right)+\left(1-q_{2}\right) u\left(y^{\frac{\theta-1}{\theta}} y_{1}^{\frac{1-9}{\theta}} y_{1}\right)-k(y)
\end{aligned}
$$

We want to look for a solution to the following two equations:

$$
\left\{\begin{array}{l}
F_{1}\left(y_{1}, y_{2}, q_{1}, q_{2}\right)=y_{1}-\bar{y}\left(y_{1}, y_{2}, q_{1}\right)=0 \\
F_{2}\left(y_{1}, y_{2}, q_{1}, q_{2}\right)=y_{2}-\dot{y}\left(y_{1}, y_{2}, q_{2}\right)=0
\end{array}\right.
$$

Let $y^{*}$ and $y^{=*}$ be two equilibrium values (such as those in figure la). Clearly at $q_{1}=$ $g_{2}=1, y^{*}$ and $y^{* \times \times}$ solve $\left(^{*}\right)$. 
We want to show that the conditions of the IFT are satisfied for $y^{*}$ and $y^{\cdots \cdots}$. If the conditions are satisfied, then for some neighbourhood around $y^{*}$ and $y^{\cdots}{ }^{\cdots}$ there will exist other values of $y$ that satisfy $\left({ }^{*}\right)$.

Thus, we are required to show that;

(i) $F_{1}$ and $F_{2}$ have continuous partial derivatives with respect to $y_{1}, y_{2}, q_{1}, q_{2}$ at $\left(y^{*}, y^{\cdots *}, 1,1\right)$.

(ii) at $\left(y^{*}, y^{\cdots \times}, 1,1\right)$ the jacobian of $\left(^{*}\right)$ does not vanish.

Condition (i) is satisfied by the assumptions on preferences. To show that condition (ii) is satisfied, note that $\bar{y}(\cdot)$ by definition solves;

$$
q_{1} u \prime\left(\frac{\theta-1}{\theta}\right] y^{\frac{-1}{\theta}} y_{1}^{\frac{1}{\theta}}+\left(1-q_{1}\right) u \prime\left[\frac{\theta-1}{\theta}\right]\left[\frac{y}{y_{1}}\right]^{\frac{-1}{\theta}} y_{2}-k \prime(y)=0
$$

Evaluating (5) at $\left(y^{*}, y^{\cdots \cdots}, 1,1\right)$, we have;

$$
\frac{\partial F_{1}}{\partial y_{1}}=\frac{[S . O . C .]-\frac{1}{\theta}\left[\frac{\theta-1}{\theta}\right] \frac{u\left(y^{*}\right)}{y^{*}} W^{\prime}\left(y^{*}\right)}{[S . O . C .]}
$$

which is strictly positive, since [S.O.C. $] \leq 0$ and $W\left(y^{*}\right)>0$ by assumption.

Furthermore, $\hat{y}$ by definition solves;

$$
q_{2} u \prime\left|\frac{\theta-1}{\theta}\right| y^{\frac{-1}{\theta}} y_{2}^{\frac{1}{\theta}}+\left(1-q_{2}\right) u \prime \mid\left[\frac{\theta-1}{\theta} \mid\left\{\frac{y}{y_{1}}\right]^{\frac{-1}{\theta}} y_{2}-k \prime(y)=0\right.
$$

Evaluating (6) at $\left(y^{*}, y^{\cdots}, 1,1\right)$ implies;

$$
\frac{\partial F_{2}}{\partial y_{2}}=\frac{[S . O . C .]-\frac{1}{\theta}\left[\frac{\theta-1}{\theta}\right] \frac{u(y \cdots)}{y^{\cdots} \cdots} W\left(y^{\cdots}\right)}{[\text { S.O.C. }]}
$$

which is also strictly positive.

Moreover, $\frac{\partial F_{1}}{\partial y_{2}}=\frac{\partial F_{2}}{\partial y_{1}}=0$ when evaluated at $\left(y^{-}, y^{\cdots \cdots}, 1,1\right)$. Hence the jacobian of $\left(^{*}\right)$ is;

$$
|J|=\frac{\partial F_{1}}{\partial y_{1}} \cdot \frac{\partial F_{2}}{\partial y_{2}}>0
$$


so that condition (ii) is satisfied.

Therefore, by the IFT, there exists a neighbourhood $N$ around $\left(y^{*}, y^{* *}, 1,1\right)$ determined by $\left|\bar{q}_{j}-1\right|<\delta_{j} ;\left|\bar{y}_{i}-y_{k}\right|<\epsilon_{i} ; \quad\left(\delta_{j}>0 ; \epsilon_{i}>0\right) \quad i=1,2 ; j=1,2 ; k=*, * * *$ such that for each $\left(\bar{q}_{1}, \bar{q}_{2}\right)$ where $\left|\bar{q}_{j}-1\right|<\delta_{j}$ there exists a unique solution $\left(\bar{y}_{1}, \bar{y}_{2}\right)$ of $\left({ }^{*}\right)$ such that $\left|\bar{y}_{i}-y_{k}\right|<\epsilon_{i}$. It is $\left(\bar{y}_{1}, \bar{y}_{2}, \bar{q}_{1}, \bar{q}_{2}\right)$ that represents an SSSE of the model.

At this stage, the existence of SSSE as randomizations between allocations in the neighborhood of two steady states has been demonstrated. However, as previously claimed, for SSSE to exist in this economy, a y such as $y^{=*}$ in figure la must exist. That is, SSSE only exist

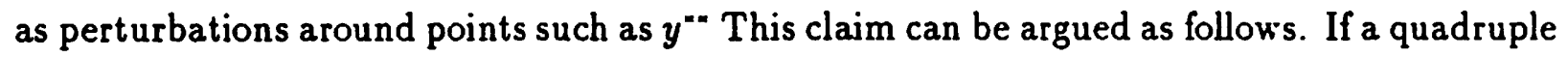
$\left(y_{1}, y_{2}, q_{1}, q_{2}\right)$ is to represent a SSSE, both $q_{1}$ and $q_{2}$ must be less than one. ${ }^{12}$ From the definition of $q_{1}$ and $q_{2}$, this would require $W\left(y_{1}\right)<K\left(y_{1}\right)$ and $W\left(y_{2}\right)>K\left(y_{2}\right)$, or equivalently, $\left[\frac{\theta-1}{\theta}\right] u \prime\left(y_{1}\right)<k \prime\left(y_{1}\right)$ and $\left[\frac{\theta-1}{\theta}\right] u \prime\left(y_{2}\right)>k \prime\left(y_{2}\right)$. Suppose $y^{*}<y_{1}<y_{2}<y^{* *}$ as is illustrated in figure 2. From figure 2 and the definition of $z(y)$, we see that at $y_{1}, W\left(y_{1}\right)<K\left(y_{1}\right)$ as required. However, $W\left(y_{2}\right)<K\left(y_{2}\right)$, which implies $q_{2}>1$. Hence, $\left(y_{1}, y_{2}, q_{1}, q_{2}\right)$ could not represent a SSSE. In fact, any $y_{2}$ such that $y^{*}<y_{2}<y^{* *}$ or $y_{2}>y^{\cdots *}$ could not be a part of a SSSE since for such a $y_{2}, q_{2}>1$. Therefore for $y_{2}$ to be a part of a SSSE, it must be that $y^{\mu *}<y_{2}<y^{\mu * *}$. By a similar argument, only $y_{1}$ such that $y^{*}<y_{1}<y^{* *}$ could be a part of a SSSE. These restrictions imply SSSE exist only as perturbations around a point such as $y^{\prime *}$. As was noted in the previous section, the presence of increasing returns to scale is required for points such as $y^{*=}$ to exist. Therefore, in this economy, the assumption of increasing returns to scale is necessary for the existence of SSSE.

Furthermore, monopolistic competition makes it more likely that $y^{=-}$will be a steady state equilibrium. Since SSSE are constructed as randomizations between multiple steady state equilibria, if $y^{=*}$ is a steady state equilibrium, then in a neighborhood around $y^{*=}$ SSSE equilibria will exist. If $y^{=-}$was not an equilibrium, the set of SSSE in the model would be confined to points in the neighborhood of $y^{*}$ and $y^{\cdots-*}$. Therefore, the presence of monopolistic 
competition expands the set of SSSE in the economy.

As a final note, it was argued in section 3 that 2 cycles would exist only if the equilibrium $y^{* *}$ was locally unstable. Since our construction of SSSE did not require any conditions on the stability of the equilibrium law of motion, it is possible for SSSE to exist in an economy for which 2 cycles do not exist. Therefore, in this model, the existence of SSSE does not depend on the existence of 2 cycles as was the case in Azariadis and Guesnerie [2].

Example: Continuing with the example of 3.1 , first note that $W(y)=.5 y^{.94}$ so that $W \prime(y)>0$; i.e. consumption and leisure are gross substitutes. To show the existence of a SSSE, let $y_{1}=.0154+\epsilon$ and $y_{2}=.4872-\epsilon$, with $\epsilon=0.01$. Then from the definitions of $q_{1}$ and $q_{2}$ we have $q_{1}=.998473$ and $q_{2}=.994634$. This then represents a SSSE. Furthermore, since both $q_{1}$ and $q_{2}$ are very close to 1 , output fluctuations will be persistent.

\section{Summary Remarks}

Within a simple overlapping generations model with money and production, it is possible to experience sunspot equilibria without requiring the assumption of gross complementarity between future consumption and present leisure. This is accomplished by introducing a monopolistically competitive product market and increasing returns to scale in the production technology to the model. The presence of monopolistic competition and increasing returns to scale allow for the possible existence of multiple steady state equilibria. Sunspot equilibria can then be constructed as randomizations around the multiple steady states. This is done without the gross complementarity requirement. It is also the case that the stationary transition probabilities may be close to one so that output fluctuations are highly persistent. Finally, the existence of 2 cycles is not necessary for the existence of SSSE in this model. 


\section{Appendix A}

This appendix derives the solution to the individual agents problem as was represented by condition (1) in the text.

The problem faced by agent $j$ at date $t$ is:

$$
\max _{\left\langle c_{t+1}^{j}: y_{i}^{j} ; n_{t}^{j} ; p_{t+1}^{j}\right\rangle} V=u\left(c_{t+1}^{j}\right)-g\left(n_{t}^{j}\right)
$$

subject to

$$
\begin{aligned}
& \text { i) } y_{t}^{j}=f\left(n_{t}^{j}\right) \\
& \text { ii) } p_{t}^{j} y_{t}^{j}-\int_{0}^{1} p_{t+1}(i) c_{t+1}^{j}(i) d i=0 \\
& \text { iii) } 0 \leq n_{t}^{j} \leq 1 \\
& \text { iv) } c_{t+1}^{j}=\left(\int_{0}^{1}\left(c_{t+1}^{j}(i)\right)^{\frac{\theta-1}{\theta}} d i\right)^{\frac{\theta}{\theta-1}}
\end{aligned}
$$

The lagrangian for this problem is then,

$$
\left.\max _{\left\langle\left(c_{t+1}^{j}(i)\right)_{n}^{1}: y_{i}^{j}\right\rangle} \Lambda=u\left[\left[\int_{0}^{1}\left(c_{t+1}^{j}(i)\right)^{\frac{\theta-1}{\theta}} d i\right]^{\frac{\theta}{\theta-1}}\right]-k\left(y_{t}^{j}\right)+\lambda_{i}^{i} p_{t}^{j} y_{t}^{j}-\int_{0}^{1} p_{t+1}(i) c_{t+1}^{j}(i) d i\right]
$$

The $\mathrm{N}+2$ first order conditions, assuming an interior solution, are thus;

$$
\begin{gathered}
u\left(\left(c_{t+1}^{j}\right)\left[\int_{0}^{1}\left(c_{t+1}^{j}(i)\right)^{\frac{\theta-1}{\theta} d i}\right]^{\frac{1}{\theta-1}}\left(c_{t+1}^{j}(i)\right)^{\frac{-1}{\theta}}=\lambda p_{t+1}(i) i \epsilon[0,1]\right. \\
k \prime\left(y_{t}^{j}\right)=\lambda\left[\frac{d p_{t}^{j}}{d y_{t}^{j}} y_{t}^{j}+p_{t}^{j}\right] \\
p_{t}^{j} y_{t}^{j}-\int_{0}^{1} p_{t+1}(i) c_{t+1}^{j}(i) d i=0
\end{gathered}
$$

Equations (A1) and (A3) imply: 


$$
c_{t+1}^{j}(k)=\frac{p_{t}^{j} y_{t}^{j}\left(p_{t+1}(k)\right)^{-\theta}}{\left(P_{t+1}\right)^{1-\theta}}
$$

for any good $k$. Noting that in a monetary equilibrium

$$
\int_{0}^{i} p_{t}^{i}(i) y_{t}^{i}(i) d i=1
$$

the supply of output by agent $\mathrm{j}$ is thus,

$$
y_{t}^{j}=\int_{0}^{1} c_{t}^{j}(i) d i=\frac{\left(p_{t}^{j}\right)^{-\theta}}{P_{t}^{1-\theta}} .
$$

From (A5) we have $\frac{d p_{i}^{j}}{d y_{\imath}^{j}}=\frac{-p_{i}^{j}}{\partial y_{i}^{j}}$. Substituting this into (A2) and solving for $\lambda$ from (A1) gives, equation (1) as outlined in the text. 


\section{Appendix B}

Appendix B supplies proofs to statements given in the text.

Proposition 2: Equation ( 3 ) evaluated at a steady state is satisfied iff $\left.\theta_{i}^{r} \varepsilon_{u \prime}-\varepsilon_{k}\right]-$ $\left[\varepsilon_{u \prime}+1\right] \leq 0$.

Proof: At a steady state (3) can be written as;

$$
u \prime \prime(y)\left[\frac{1-\theta}{\theta}\right]^{2}+\frac{u \prime(y)}{\theta y}\left[\frac{1-\theta}{\theta}\right]-k \prime \prime(y) \leq 0
$$

Furthermore, at a steady state $u \prime(y)\left[\frac{\theta-1}{\theta}\right]=k \prime(y)$ so that (B1) can be rewritten as;

$$
\frac{-k \prime(y)}{y \theta}\left[\frac{u \prime \prime(y) y}{u \prime(y)}(1-\theta)+1\right]-k \prime \prime(y) \leq 0 .
$$

But (B2) can be written as;

$$
\frac{k^{\prime}(y)}{y}\left[-\frac{1}{\theta}\left[\frac{u \prime \prime(y) y}{u \prime(y)}(1-\theta)+1\right]-\frac{k \prime \prime(y) y}{k \prime(y)}\right] \leq 0 .
$$

By definition we have $\varepsilon_{u \prime}=\frac{u \prime(y) y}{u(y)}$ and $\varepsilon_{k}=\frac{k \prime \prime(y) y}{k \prime(y)}$. Thus, (B3) is true iff

$$
-\frac{1}{\theta}\left[\varepsilon_{u}(1-\theta)+1\right]-\varepsilon_{k} \leq 0
$$

which when rearranged implies $\theta\left[\varepsilon_{u \prime}-\varepsilon_{k}\right]-\left[\varepsilon_{u \prime}+1\right] \leq 0$ as required. Q.E.D. 


\section{References}

[1] R. Aumann, J. Peck, K. Shell, "Asymmetric Information and Sunspot Equilibria: A Family of Simple Examples.", In Models of Incomplete Information and Bounded Rationality forthcoming ed. H.W. Kuhn, New York: Springer-Verlag.

[2] C. Azariadis, "Self-fulfilling Prophecies", Journal of Economic Theory 25 (1981), 380. 396.

[3] C. Azariadis and R. Guesnerie, "Sunspots and Cycles", Review of Economic Studies 53 (1986), 725-737.

[4] O. Blanchard and N. Kiyotaki, "Monopolistic Competition and the Effects of Aggregate Demand." American Economic Review 77 (1987), 647-666.

[5] D. Cass and K. Shell, "Do Sunspots Matter?", Journal of Political Economy 91 (1983), 193-227.

[6] J. Campbell and N.G. Mankiw, "Are Output Fluctuations Transitory?", Quarterly Journal of Economics C11 (1987), 857-880.

[7] S. Chatterjee R. Cooper and B. Ravikumar, "Participation Dynamics: Sunspots and Cycles", 1990 Meeting of the WEA, 1990.

[8] R. Cooper and A. John, "Coordinating Coordination Failures in Keynesian Models", Quarterly Journal of Economics 103 (1988), 441-464.

[9] P. Howitt, "Determinate Outcomes with Multiple Equilibria," University of Western Ontario, mimeo, 1990.

[10] P. Howitt and P.R. McAfee, "Animal Spirits", University of Western Ontario, research paper, 1990. 
[11] M. Killingsworth, Labour Supply, Cambridge University Press, New York 1983.

[12] T.J. Kehoe, D.K. Levine, and P.M. Romer, "Determinacy of Equilibria in Dynamic Models with Finitely Many Consumers", Journal of Economic Theory, 50 (1990), 1-21.

[13] J. Peck and K. Shell, "Market Uncertainty: Correlated and Sunspot Equilibria in Imperfectly Competitive Economies." Review of Economic Studies 58 (1991), 1011-1029.

[14] P. Phillips, "To Criticise The Critics: An Objective Bayesian Analysis of Stochastic Trends," Forthcoming, Journal of Applied Econometrics (1990)

[15] K. Shell, "Monnaie et Allocation Intertemporelle", CNRS Seminaire d'econometrie de M. Edmond Malinvaud 21 November 1977.

[16] K. Shell, "Sunspot Equilibria", in The New Palgrave: A Dictionary of Economics (J. Eatwell, M. Milgate, P. Newman Eds.), Vol 4, 1987.

[17] K. Shell and R. Wright, "Indivisibilities: Lotteries and Sunspot Equilibria." Economic Theory, forthcoming.

[18] S. Spear,"Sufficient Conditions for the Existence of Sunspot Equilibria", Journal of Economic Theory, 34 (1984), 360-370.

[19] S. Spear, "Growth, Externalities, and Sunspots." Journal of Economic Theory 54 (1991), 215-223.

[20] P. Weil, "Increasing Returns and Animal Spirits," American Economic Review, 79 (1990), 889-894.

[21] M. Woodford, "Stationary Sunspot Equilibria in a Finance Constrained Economy", Journal of Economic Theory 40 (1986), 128-137. 


\section{Footnotes}

1. See Killingsworth [11] for a summary of such studies.

2. As in Blanchard and Kiyotaki [4], $\theta>1$ is required to generate an equilibrium.

3. It is basically assumed that $f^{-1}(y)$ has the same properties as $u(y)$. This assumption is satisfied fora general class of functions. For instance $f^{-1}(y)=y^{a} ; 0 \leq a \leq 1$ satisfies assumption 1 .

4. The proof of this statement is given in appendix B.

5. In this model, future consumption and current leisure are gross substitutes if $-\frac{d n_{1}}{d p_{t+1}}>$ 0 . But $-\frac{d n_{t}}{d p_{t+1}}=\frac{d f^{-1}\left(y_{t}\right)}{d y_{t}} \frac{d y_{t}}{d p_{t+1}}>0$ iff $1+\varepsilon_{u}>0$.

6. For $y^{*}, y^{-\infty}$, and $y^{\mu-\cdots}$ to be equilibria, their respective second order conditions must also be satisfied. For $y^{*}$ and $y^{-\cdots}$ this is trivially true. This follows from the fact that $z \prime(y)<0$ at $y^{*}$ and $y^{\cdots}$ so that $\varepsilon_{u}<\varepsilon_{k}$ and hence equation 4 is satisfied. For $y_{\Sigma-} \varepsilon_{u}>\varepsilon_{k}$ so that equation 4 may not be satisfied for all $y$.

7. While proposition 1 gives a sufficient condition for multiple equilibria, it also represents a necessary condition if ii) is rewritten with a weak inequality. This distinction must be made so as to rule out the possibility of $z(y)=0$ and $\varepsilon_{u}=\varepsilon_{k}$ with $y$ being a point of inflection. This case is illustrated in figure $1 \mathrm{~b}$.

8. It is possible that $\varepsilon_{u}(y)=\varepsilon_{k}(y)$ at $y^{* *}$ so that the second order condition is satisfied and $y^{* *}$ is an equilibrium.

9. Note, $\varepsilon_{k}=\varepsilon_{g}+\varepsilon_{f-1}$. If $f^{-1}(y)$ is of the form $y^{\alpha}$ with $0<\alpha<1$, then $\varepsilon_{f-1}=(\alpha-1)>$ -1 . But since $\varepsilon_{g}>0, \quad \varepsilon_{k}>-1$ implying equilibria such as $y *=$ would always be locally stable.

10. The fact that the steady state equilibria can be Pareto ranked is consistent of all models with strategic complementarities and spillovers. For a more detailed discussion see Cooper and John [8].

11. This is equivalent to the assumption that $\varepsilon_{u}>-1$ as was stated previously. 
12. This is excluding the trivial SSSE for which $q_{1}=q_{2}=1$ and $y_{1}$ and $y_{2}$ are steady state equilibria. 


\section{Figure 1a}

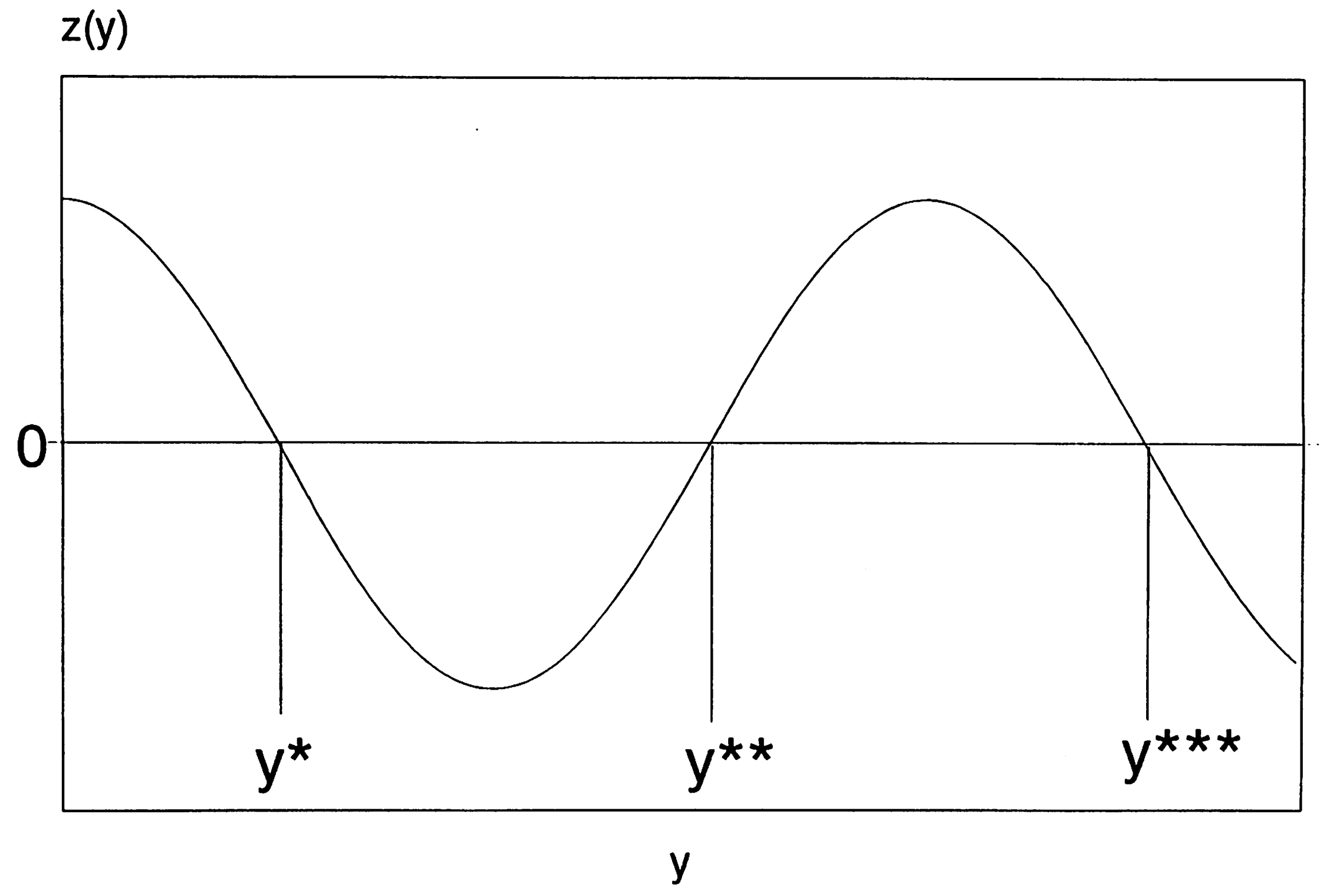




\section{Figure $1 b$}

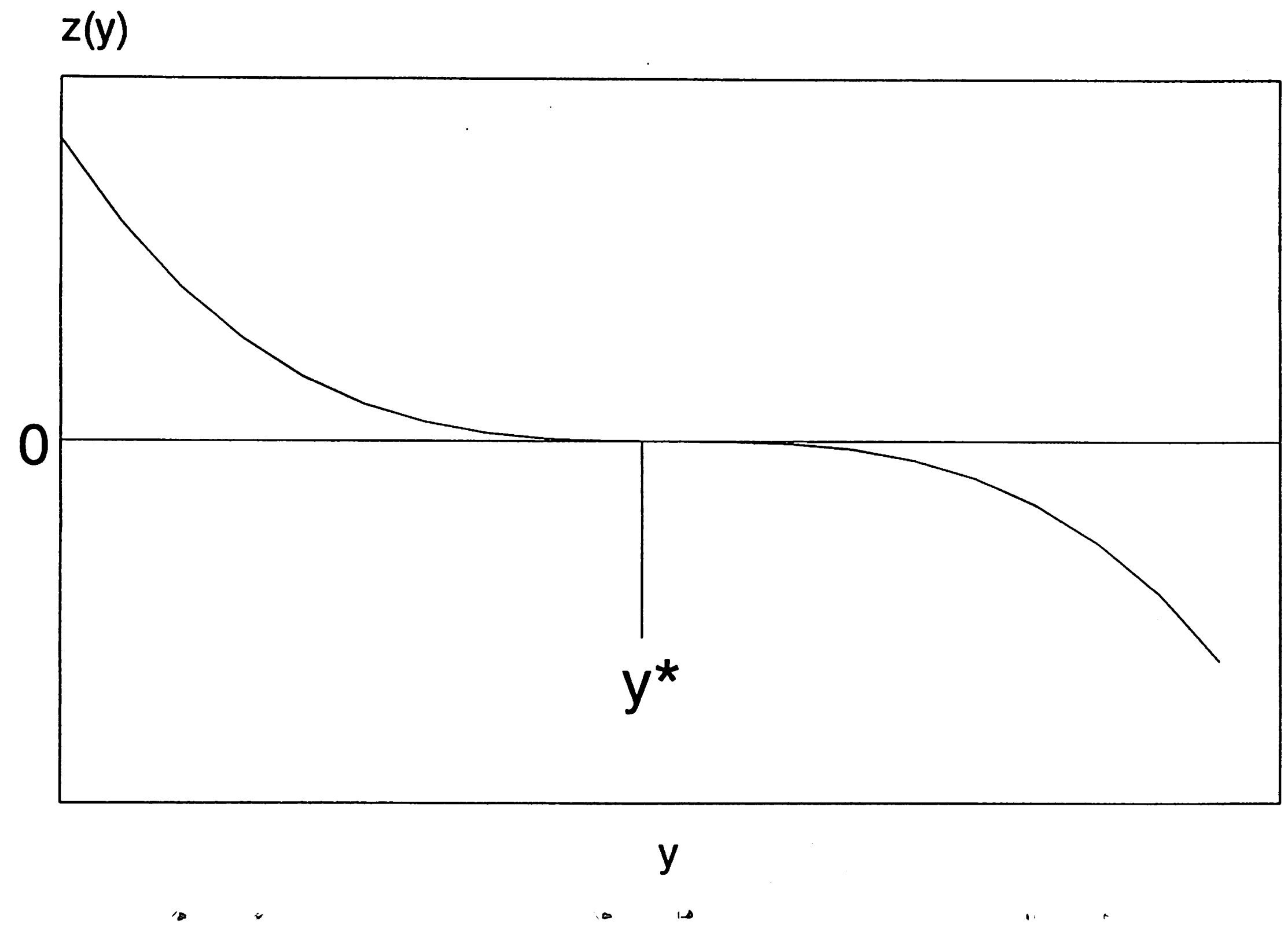




\section{Figure 2}

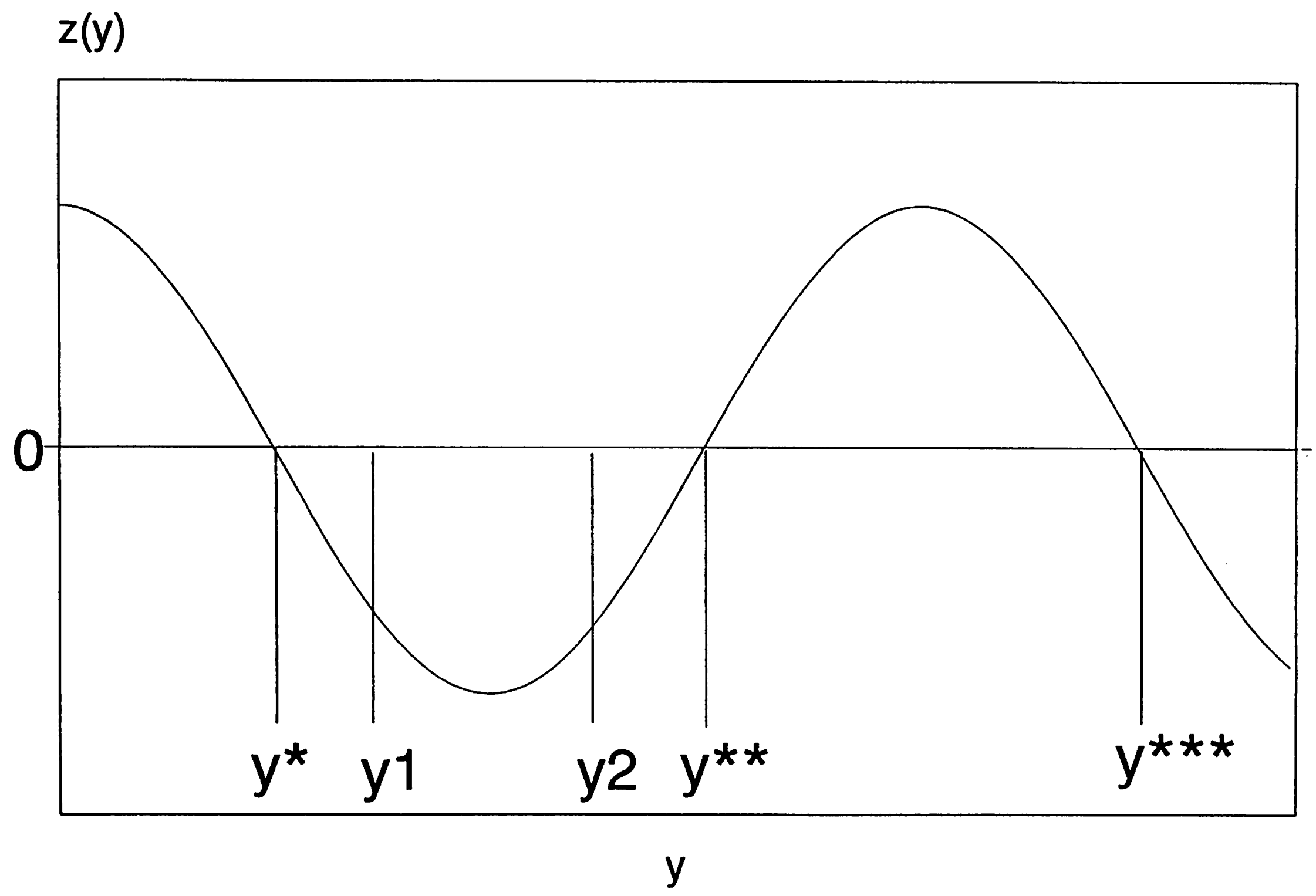

